Trauma Berufskrankh 2006 • 8 [Suppl 1]: S40-S43 DOI 10.1007/s10039-006-1116-0

Online publiziert: 15. März 2006

(c) Springer Medizin Verlag 2006

R. Klose

Abteilung für Anästhesie, Intensivmedizin und

Schmerztherapie, BG-Unfallklinik, Ludwigshafen

\title{
Schmerztherapeutische Versorgungsstrategien
}

\section{Pathogenese}

Zur Frage, warum ein Akutschmerz in einen chronischen Schmerz übergeht, sind von der Schmerzforschung in den vergangenen Jahrzehnten bemerkenswerte Ergebnisse geliefert worden $[12,16,17]$. Auf sehr komplexe Weise kann ein akuter Schmerzreiz, wenn er anhaltend oder wiederkehrend ist, auf den verschiedenen Ebenen der Impulsverarbeitung (Peripherie, Rückenmark, Gehirn) zu einer Reizimpulsverstärkung und zur Hinterlassung von "Schmerzspuren“ führen (• Abb. 1). Aufgrund der neuronalen Plastizität kann sich rasch ein so genanntes "Schmerzgedächtnis" entwickeln. Schließlich ist der Schmerz auch dann vorhanden, wenn die primär auslösende Ursache $-z$. B. Verletzung, Operationswunde - nicht mehr besteht [19].

Am Chronifizierungsprozess haben neben dem initial primären Schmerz weitere Faktoren wesentlichen Anteil:

- Unzureichende Behandlung des Akutschmerzes
- Genetische Veranlagung (Risikogruppe?)

- Psychische Einflüsse Psychiatrische Erkrankungen (Depression) Problem- und Stressbewältigungsstrategien

- Soziale Faktoren Familiäres Umfeld Berufliche Situation Alter

Die Inzidenz chronischer Nervenschmerzen nach Operationen ist auch unter Beachtung definierter Kriterien [9] nicht gering. Diese Kriterien sind:

- Der Schmerz entwickelt sich nach einer Operation.

- Der Schmerz besteht mindestens 2 Monate.

- Es ist keine andere Ursache für den Schmerz erkennbar.

- Der postoperative Schmerz ist nicht die Fortsetzung eines präoperativen Schmerzes. ger als 6 Monate anhaltender oder wiederkehrender Schmerz - leiden in Deutschland etwa 20 Mio. Menschen, 5-8 Mio. erfahren dadurch erhebliche Einschränkungen und etwa 500.000-800.000 (10\%) müssen als Problemfälle mit einer schwer zu behandelnden Schmerzkrankheit eingestuft werden. Die jährlichen Kosten für Schmerzerkrankungen sind in der Bundesrepublik mit 20,5-28,7 Mrd. EUR erheblich. Auf die direkten Kosten (Behandlung, Medikamente, Rehabilitation) entfallen etwa 12.000-16.00o Mrd. EUR, auf die indirekten Kosten (Arbeitsunfähigkeit, vorzeitige Berentung) 9000-13.000 Mrd. EUR [12].

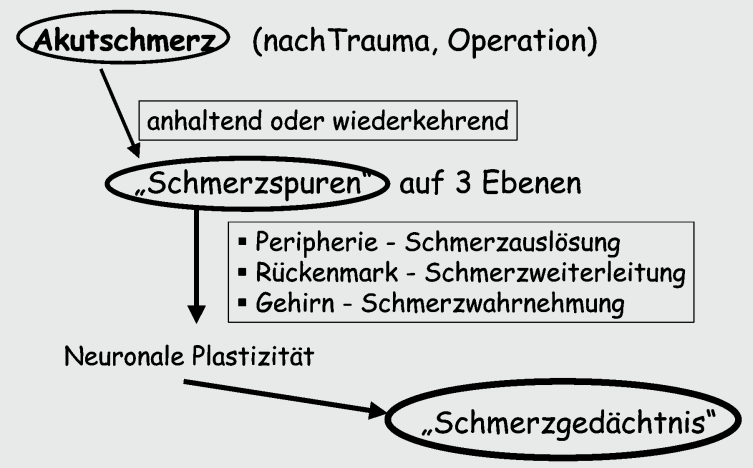

Abb. 14 Chronifizierung von Schmerz über die 3 Ebenen der Schmerzverarbeitung 
Im Mittel muss mit einem Auftreten in bis $\mathrm{zu} 35 \%$ der Fälle gerechnet werden (- Tab. 1). Bemerkenswert ist, dass auch die modernen minimalinvasiven Eingriffe nicht zu einer überzeugenden Reduktion dieser postoperativen Komplikation beitragen, obgleich sicherlich die Operationstechnik mit entsprechender Schnittführung und Schonung von Nerven einen Einfluss hat $[1,4,8]$.

\section{Prävention}

Bei der Suche nach prädiktiven Faktoren für eine mögliche Schmerzchronifizierung gerät immer wieder der starke Akutschmerz in den Mittelpunkt. Daraus folgt, dass eine konsequente und effiziente Akutschmerztherapie zu fordern ist (• Tab. 2). Über den humanitären Aspekt, Leiden zu mildern, hinaus hat eine solche Schmerzbehandlung belegte positive Effekte sowohl durch Stressreduktion auf den unmittelbaren postoperativen bzw. posttraumatischen Verlauf als auch durch verbesserte Rehabilitationschancen einen anhaltenden Langzeiteffekt.

Unter der Prämisse, dass die Chronifizierung mit dem Trauma, d. h. auch mit dem Hautschnitt, beginnt, erscheint die Frage nach einer präemptiven bzw. präventiven Analgesie durchaus gerechtfertigt. Legt man als Zielparameter postoperativ einen späteren und geringeren Arzneimittelverbrauch zugrunde, kann den Regionalanästhesieverfahren, insbesondere der Epiduralanästhesie, der lokalen Wundinfiltration, aber auch einer präoperativen Gabe von NSAID ein präventiver analgetischer Effekt zugestanden werden [13]

Für das Fachgebiet der Traumatologie ist die Reduktion des Phantomschmerzes nach Amputationen durch eine frühzeitige Regionalanästhesie bzw. durch die Gabe von Ketamin bedeutsam [3]. Zunehmend gewinnt diese Substanz in der Behandlung des chronischen Schmerzes Bedeutung [6].

Rückenmarknahe oder periphere Nervenblockaden zur intraoperativen Schmerzausschaltung und ihre konsequente Fortführung als Katheterverfahren zur postoperativen Analgesie bieten erhebliche Vorteile (• Tab. 3) und sind in der Lage, über die Operationsphase hi-

Trauma Berufskrankh 2006 · 8 [Suppl 1]: S40-S43 DOI 10.1007/s10039-006-1116-0

(c) Springer Medizin Verlag 2006

\section{R. Klose}

\section{Schmerztherapeutische Versorgungsstrategien}

\section{Zusammenfassung}

Akuter Schmerz ist für das Überleben notwendig, stellt er doch ein Warnsymptom dar. Chronische Schmerzen dagegen sind als Krankheit zu betrachten, von der in Deutschland etwa 20 Mio. Menschen betroffen sind. Sie gehen mit erheblichen Behandlungs- und Folgekosten einher. Nach Operationen treten sie in bis zu 35\% der Fälle auf, diese Rate konnte auch mit minimalinvasiven Verfahren nicht entscheidend gesenkt werden. Bei der Chronifizierung spielt anscheinend der starke Akutschmerz eine bedeutende Rolle, weswegen eine konsequente und effiziente Akutschmerztherapie zu fordern ist. Hierfür kommen medikamentöse Analgesie

\section{Care strategies in pain therapy}

\section{Abstract}

Acute pain is necessary for survival; it is, after all, a warning. Chronic pain, in contrast, has to be regarded as an illness; it affects about 20 million people in Germany and its treatment and later consequences involve substantial costs. After operations about $35 \%$ of patients experience chronic pain, and it has not proved possible to lower this rate even with minimally invasive procedures. Severe acute pain seems to have a significant factor in chronification, which is why it requires consistent and effective short-term treatment. Medicamentous analgesia and anaesthesia, und Anästhesie, rückenmarknahe oder periphere Nervenblockaden in Frage. Da diese Maßnahmen z. T. mit lebensbedrohlichen Komplikationen einhergehen können, sind zur Risikominderung eine klare und verbindliche Schmerztherapieleitlinie und ein Akutschmerzdienst zu fordern, der jederzeit zur Verfügung steht.

\section{Schlüsselwörter}

Akuter Schmerz - Chronischer Schmerz . Konsequente und effiziente Akutschmerztherapie · Schmerztherapieleitlinien · Akutschmerzdienst

with nerve blocks in the vicinity of the spinal cord or in peripheral regions, can be considered for this purpose. Since these measures can be associated with life-threatening complications in some circumstances, a clear and binding guideline on pain therapy should be required, as should an acute pain service that is available round the clock.

\section{Keywords}

Acute pain - Chronic pain - Consistent and effective therapy for acute pain - Guidlines on pain therapy · Acute pain service 


\section{Schmerztherapie nach Trauma}

Tab. 1 Chronische Schmerzen nach operativen Eingriffen

\begin{tabular}{|ll} 
Schmerzen & Häufigkeit [\%] \\
Phantomschmerzen nach Amputationen & 60 \\
\hline „postthoracotomy pain syndrome“ & 50 \\
\hline Videoassistierte Thorakotomie! & 63 \\
\hline ACVB (Sternotomie) & 56 \\
\hline Brustchirurgie & 30 \\
\hline Gallenblasenchirurgie & 20 \\
\hline V.-saphena-Exstirpation & 4 \\
\hline Leistenbruchchirurgie & 10 \\
\hline Laparoskopisch! & 6 \\
\hline Zahnextraktion (3. Molar) & 23 \\
Nach Aasvang u. Kehlet [1], Eisenberg [4], Macrae [9], Perkins u. Gopal [14], Perkins u. Kehlet [15]
\end{tabular}

Tab. 2 Akutschmerzbehandlung

\begin{tabular}{|c|c|c|}
\hline Aspekt & Symptome & Zweck \\
\hline Humanitärer Aspekt & & Leidensreduktion \\
\hline \multirow[t]{13}{*}{ Physiologischer Aspekt } & Erhöhter Blutdruck & \multirow[t]{13}{*}{ Stressreduktion } \\
\hline & Erhöhte Herzarbeit & \\
\hline & Erhöhtes Infarktrisiko & \\
\hline & Erhöhter Blutzucker & \\
\hline & Ileus & \\
\hline & Schlechte Wundheilung & \\
\hline & $\begin{array}{l}\text { Schlechte immunologische } \\
\text { Abwehr }\end{array}$ & \\
\hline & Kognitive Störungen & \\
\hline & Delir & \\
\hline & Schlafstörungen & \\
\hline & Pulmonale Komplikationen & \\
\hline & Verzögerte Rekonvaleszenz & \\
\hline & Verlängerter Klinikaufenthalt & \\
\hline
\end{tabular}

Tab. 3 Vorteile regionaler Anästhesie-/Analgesieverfahren

\section{Erkrankung}

Magen-Darm-Atonie

Myokardinfarkt

Kardiovaskuläre Komplikationen

Pulmonale Infekte

Postoperative Beatmungsdauer

Chirurgische Komplikationen

Stationäre Verweildauer

Erfüllung von Entlassungskriterien

Krankenhauskosten

Bezugspunkte sind die jeweiligen Werte nach Allgemeinanästhesie bzw. systemischen Anlagetika, nach Jage et al. [7]

naus langfristig Schmerzen, d. h. Chronifizierungsprozesse, zu verhindern - z. B. Epiduralanästhesie und Postthorakotomiesyndrom. Nach unfallchirurgisch-orthopädischen Eingriffen lassen sich mit kontinuierlichen Nervenblockaden deut-

\section{Verbesserung}

Etwa 2 Tage kürzere Behandlungsdauer Etwa $30 \%$ geringeres Auftreten

Etwa $75 \%$ geringeres Auftreten Etwa $30 \%$ geringeres Auftreten Etwa 40\% kürzere Behandlungsdauer Etwa 50\% geringeres Auftreten Etwa 30-50\% kürzer

Etwa 25-30\% eher

Etwa 20-50\% geringe schwerste lebensbedrohende Nebenwirkungen möglich sind:

- Benommenheit

- Müdigkeit

- Übelkeit

- Erbrechen

- Atemdepression

- Atemstillstand

- Obstipation

- Magengeschwür

- Magenblutung

- Magendurchbruch

- Nierenschäden

- Nervenschäden

- Nervenschmerzen

- (Querschnitt)lähmungen

- Pneumothorax

- Infektionen

- Blutungen usw.

Sollen die Vorteile einer guten postoperativen Analgesie voll genutzt werden, sind zur Risikominderung eine klare und verbindliche Schmerztherapieleitlinie interdisziplinär zu erstellen und ein Akutschmerzdienst zu fordern, der jederzeit - auch nachts und am Wochenende - zur Verfügung steht. Im Rahmen eines umfassenden Qualitätsmanagements hat dieser auch Aufgaben in der Dokumentation von Nebenwirkungen, Schulung und Überwachung [20]. Hilfestellung bei der Etablierung eines solchen Akutschmerzdienstes leisten die vom Arbeitskreis Akutschmerz der DGSS herausgegebenen Empfehlungen [11].

Der Verdacht auf eine drohende oder bereits eingetretene Chronifizierung des Schmerzes besteht bei:

- Schmerz, der über die erwartete normale Heilungszeit hinausgeht (Faustregel: Akutschmerz selten $>1$ Monat)

- Änderung des Schmerzcharakters

- Schmerz ohne Warnfunktion

- Schmerzen nicht nur im Verletzungsbereich, Ausbreitung der Schmerzen

- Allgemeine körperliche und seelische Befindungsstörungen

- Häufiger, zunehmender, ständiger Gebrauch von Schmerz- und Beruhigungsmitteln

lich bessere Rehabilitationsergebnisse erzielen $[1,18]$.

Die positiven Ergebnisse einer guten und effizienten postoperativen/posttraumatischen Schmerztherapie dürfen aber nicht darüber hinwegtäuschen, dass auch
In diesen Fällen sollte der Patient umgehend in einer schmerztherapeutischen Einrichtung vorgestellt werden. Diese muss eine interdisziplinäre multimoda- 
le Schmerztherapie, bei der Schmerztherapeut, Psychologe, Physiotherapeut, Ergotherapeut, Sozialarbeiter u. a. gleichberechtigt mitarbeiten, anbieten. Nur ein solches Behandlungskonzept wird dem chronischen Schmerz als biopsychosoziale Schmerzkrankheit gerecht.

\section{Fazit}

In der BG-Unfallklinik Ludwigshafen hat sich eine ganztägige ambulante interdisziplinäre Schmerzdiagnostik unter Einbezug der oben genannten Fachgruppen bewährt, um den Berufsgenossenschaften für ein anstehendes Heilverfahren Empfehlungen an die Hand zu geben. Darüber hinaus sehen wir in einem 4-wöchigen schmerztherapeutischen Intensivprogramm eine sinnvolle Ergänzung der bereits bestehenden Behandlungsoptionen. Dieses interdisziplinäre, teilstationäre Programm in einer Tagesklinik umfasst standardisierte Gruppensitzungen, die von individuellen Einzelsitzungen ergänzt werden. Dabei wird der Schwerpunkt auf übende, mittelund langfristig wirkende Maßnahmen gelegt. Strategie ist, den Patienten verantwortlich und aktiv in die Bewältigung seines Schmerzes einzubinden. Mit zunehmender Kompetenz des Patienten kann und soll sich das Behandlerteam zurückziehen.

\section{Korrespondierender Autor}

\section{Prof. Dr. R. Klose}

Abteilung für Anästhesie, Intensivmedizin und Schmerztherapie, BG-Unfallklinik Ludwig-Guttmann-Straße 13, 67071 Ludwigshafen

klose@bgu-ludwigshafen.de

Interessenkonflikt. Es besteht kein Interessenkonflikt. Der korrespondierende Autor versichert, dass keine Verbindungen mit einer Firma, deren Produkt in dem Artikel genannt ist, oder einer Firma, die ein Konkurrenzprodukt vertreibt, bestehen. Die Präsentation des Themas ist unabhängig und die Darstellung der Inhalte produktneutral.

\section{Literatur}

1. Aasvang $\mathrm{E}$, Kehlet $\mathrm{H}$ (2005) Chronic postoperative pain: the case of inguinal herniorrhaphy. $\mathrm{Br} J$ Anesth 95: 69-76
2. Capdevila X, Barthelet Y, Biboulet P et al. (1999) Effects of perioperative analgesic technique on the surgical outcome and duration of rehabilitation after major knee surgery. Anesthesiology 91: 8-15

3. Dertwinkel R, Heinrichs C, Senne I et al. (2002) Prevention of severe phantom limb pain by perioperative administration of ketamine - an observational study. Acute Pain 4: 9-13

4. Eisenberg E (2004) Post-surgical neuralgia. Pain 111:3-7

5. Gerbershagen U (1986) Organisierte Schmerzbehandlung - Eine Standortbestimmung. Internist 27: 459-469

6. Hocking G, Cousins MJ (2003) Ketamine in chronic pain management: an evidence-based review. Anesth Analg 97: 1730-1739

7. Jage J, Tryba M, Neugebauer E et al. (2005) Postoperative Schmerztherapie - eine interdisziplinäre Notwendigkeit. Dtsch Ärztebl 102: A 361-366

8. Landreneau RJ, Mack MJ, Hazelrigg SR et al. (1994) Prevalence of chronic pain after pulmonary resection by thoracotomy or video-assisted thoracic surgery. J Thorac Cardiovasc Surg 107: 1079-1086

9. Macrae WA (2001) Chronic pain after surgery. Br J Anaesth 87: 88-98

10. Nagasako EM, Oaklander AL, Dworkin RH (2003) Congenital insensitivity to pain: an update. Pain 101: 213-219

11. Neugebauer E, Wiebalck A, Stehr-Zirngibl S (Hrsg) (2003) Akutschmerztherapie - ein Curriculum für Chirurgen. Unimed, Bremen

12. Niesert W, Zenz M (2005) Prophylaxe chronischer Schmerzen. Dtsch Ärztebl 102: A1586-1985

13. Ong CKS, Lirk Ph, Seymour RA et al. (2005) The efficacy of preemptive analgesia for acute postoperative pain management: a meta-analysis. Anesth Analg 100: 757-773

14. Perkins FW, Gopal A (2003) Postsurgical chronic pain. Tech Reg Anesth Pain Manage 7: 122-126

15. Perkins FW, Kehlet $\mathrm{H}$ (2000) Chronic pain as an outcome of surgery. Anesthesiology 93: 11231133

16. Sandkühler J (2001) Schmerzgedächtnis. Dtsch Ärztebl 98: A 2725-2730

17. Siddall PJ, Cousins MJ (2004) Persistent pain as a disease entity: implications for clinical management. Anesth Analg 99: 510-520

18. Singelyn FJ, Gouverneur JMA (1999) Postoperative analgesia after total hip arthroplasty: i.v. PCA with morphine, patient-controlled epidural analgesia, or continous "3-in-1-block". A prospective evaluation by our acute pain service in more than 1,300 patients. J Clin Anesth 11:550-554

19. Woolf CJ, Costigan M (2000) Neuronal plasticity: increasing the gain in pain. Science 288: 17651769

20. Wulf H, Neugebauer E, Maier Ch (Hrsg) (1997) Die Behandlung akuter perioperativer und posttraumatischer Schmerzen. Empfehlungen einer interdisziplinären Expertenkommission. Thieme, Stuttgart New York 